\title{
Méthode rapide des composés très volatils de l'arôme des produits laitiers Application au yoghourt
}

\author{
par \\ J. P. DUMONT et J. ADDA \\ Laboratoire d'Etude des Arômes, Station Centrale de Recherches \\ Laitières et de Technologie des Produits Animaux \\ I.N.R.A. (78) Jouy-en-Josas
}

\section{PRESENTATION}

Parmi tous les procédés utilisés pour isoler les corps impliqués dans l'arôme des produits naturels, les méthodes destinées à étudier les composés très volatils responsables de l'odeur occupent une place de choix $[1,2]$. En effet, ces techniques allient généralement la rapidité avec l'aptitude à mettre en évidence les proportions relatives de substances qui sont très souvent sévèrement affectées par les procédés classiques de récupération des produits d'arôme. La pratique désormais classique dite du " Head Space » se trouve être cependant limitée dans certains cas par la faible concentration des produits à étudier, aussi, plutôt que d'avoir recours à des " équilibrages " de l'échantillon à des températures trop élevées, certains auteurs ont préféré procéder à un enrichissement de l'atmosphère gazeuse avant l'analyse [3]. Par ailleurs, d'autres chercheurs tels Morgan [4] ont substitué une méthode dynamique à l'équilibrage statique qui est le fait du " Head Space".

Désireux de compléter la gamme des techniques précédemment décrites [5], il nous a semblé indispensable de mettre au point une méthode adaptée à l'étude des substances très volatiles des produits laitiers. Après une série d'essais préliminaires, il est apparu que ce procédé convenait tout particulièrement à l'étude du yoghourt dans lequel il permet une détection aisée de l'acétaldéhyde. Quoiqu'il soit établi depuis fort longtemps que l'acétaldéhyde est le constituant majeur, quantitativement, du yoghourt fait avec du lait de vache [6], certains travaux [7] effectués sur du yoghourt fabriqué à partir de lait de brebis (comme cela est usuel dans les Balkans) [8] incitent à douter de son importance organoleptique. L'importance prise par les laits fermentés dans la production laitière française nous a semblé être une raison suffisante de tenter d'éclaircir ce problème. 


\section{MATERIEL ET METHODES}

\section{A. - Obtention des composés volatils}

1) « Appareillage »

Le système utilisé comporte deux éléments principaux qui sont :

- le barbotteur en verre dans lequel un courant d'azote «purge» l'échantillon de ses éléments volatils,

- le piège dans lequel ces derniers sont condensés.

Le barbotteur : Dans le cas où l'échantillon est un liquide même relativement visqueux (le yoghourt constitue un cas limite) on utilise un tube laveur de Maquenne à spirale moulée d'une contenance de $25 \mathrm{ml}$ (fig. 1). La prise d'essai est alors de l'ordre de 5 à $6 \mathrm{ml}$ de liquide auxquels on ajoute environ $2 \mathrm{~g}$ de $\mathrm{Na}_{2} \mathrm{SO}_{4}$ anhydre. Lorsque l'échantillon est un liquide extrêmement visqueux ou un solide (fromage), le barbotteur peut être remplacé par un flacon laveur à verre fritté (Sovirel no 4 832-54).



fig. 1

Système utilisé pour isoler les substances très volatiles d'un échantillon liquide

Le piège : Il est fait d'un tube en acier inox de $1 / 8$ " de diamètre extérieur façonné en forme de "U " et muni à ses extrémités de raccords Swagelok. L'une de ses extrémités présente une boucle afin d'éviter la condensation trop localisée de la vapeur d'eau lors du refroidissement tandis que l'autre est munie d'une vanne Nupro.

Le barbotteur est relié au piège par le moyen d'un tube téflon $1 / 8$ " extrêmement court. 


\section{2) Provenance des échantillons}

Les yoghourts utilisés dans cette étude ont été fabriqués à la Laiterie expérimentale de Jouy avec des suspensions concentrées de souches de Streptococcus thermophilus Sy 126 et Lactobacillus Bulgaricus Ly 126 ajoutées à raison de $5 \mathrm{~g} / 1$ soit dans du lait de brebis préalablement chauffé à $90^{\circ} \mathrm{C}$ pendant $10 \mathrm{mn}$, soit dans du lait de vache homogénéisé ayant subi un traitement U.H.T. (135 $\mathrm{C} 10 \mathrm{~s})$.

Le lait de vache contenait $11 \mathrm{~g} / 1$ de matière grasse et avait été enrichi par de la poudre de lait écrémé jusqu'à atteindre un E.S. de $125 \mathrm{~g} / \mathrm{kg}$.

Dans les deux cas, les laits ensemencés ont été incubés à $45^{\circ} \mathrm{C}$ pendant $3 \mathrm{~h}$.

\section{3) RÉCUPÉRATION DES SUBSTANCES VOLATILES}

L'échantillon est placé dans le barbotteur qui est ensuite connecté au piège. On permet alors l'arrivée de l'azote tandis que l'on plonge dans l'azote liquide l'extrémité inférieure du piège dont la vanne a été fermée (afin d'éviter l'aspiration de vapeurs provenant du laboratoire). Il faut prendre soin de laisser la boucle nettement audessus du niveau du liquide réfrigérant afin d'éviter la formation d'un bouchon de glace qui entraverait le passage de l'azote. Après équilibrage des pressions, la vanne du piège est ouverte et le barbottage se poursuit pendant $1 \mathrm{~h}$ avec un débit d'azote de l'ordre de 25 à $30 \mathrm{ml} / \mathrm{mn}$.

\section{B. - Analyse}

L'analyse est effectuée par chromatographie en phase gazeuse.

Réinjection : Lorsque le temps de barbottage est écoulé, le courant d'azote est interrompu et la vanne du piège est fermée, ce dernier, maintenu dans l'azote liquide, est alors séparé du restant du système et aussitôt connecté à l'injecteur du chromatographe modifié par nos soins (fig. 2), l'extrémité libre de la vanne est reliée à l'arrivée de gaz vecteur au moyen d'une tubulure souple. Un bécher rempli d'eau bouillante est substitué au Dewar contenant l'azote liquide. Après une période de l'ordre de 20 à $30 \mathrm{~s}$, la vanne est ouverte ce qui permet au gaz vecteur de balayer le piège et de réaliser ainsi une injection quasiment instantanée.

Matériel de chromatographie : On utilise un appareil Girdel (75 CD PT), les colonnes sont en acier inoxydable de diamètre extérieur $1 / 8$ de pouce $(3,2 \mathrm{~mm})$ : la colonne " 1 ", d'une longueur de $4 \mathrm{~m}$ est remplie de chromosorb W 60/80 imprégné à 20 p. 100 par du 1.2.3. Tricyanoéthoxypropane tandis que la colonne " 2 ", longue de $3 \mathrm{~m}$ est remplie de Porapak Q 80/100 mesh. La température du four dont le taux de montée est de $1,5^{\circ} \mathrm{C}$ par mn est programmée de 25 à 
$110^{\circ} \mathrm{C}$ lors des analyses sur la colonne 1 et de 100 à $170^{\circ} \mathrm{C}$ lorsque la colonne 2 est utilisée. Le gaz vecteur est l'azote et son débit est de $15 \mathrm{ml}$ par mn.

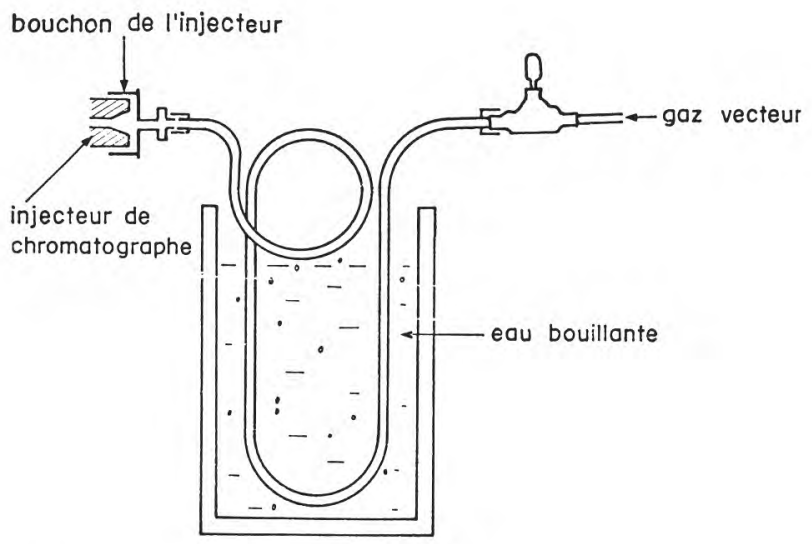

fig. 2

Injection dans le chromatographe des corps récupérés

\section{RESULTATS}

\section{A. - Essai de détermination de l'efficacité de la méthode à l'aide de solutions synthétiques}

Plusieurs composés qui figurent parmi ceux déjà répertoriés dans le yoghourt [9] ont été choisis comme témoins, mis en solution aqueuse à des concentrations de l'ordre de 5 p.p.m. La dilution obtenue a été soumise au barbottage. Les pourcentages de récupération sont présentés au tableau 1.

TABLEAU 1

\begin{tabular}{l|c}
\hline Composé témoin & Récupération (p. 100) \\
\hline Acétaldéhyde & 92 \\
Acétone & 75 \\
Ethanol & 39 \\
Diacétyle & 88 \\
\hline
\end{tabular}

La sensibilité de la méthode apparaît essentiellement limitée par celle du chromatographe. 


\section{B. - Application au yoghourt - comparaison des profils}

Les premiers essais effectués sur le yoghourt entier ont posé un problème de nettoyage du barbotteur. Aussi, compte tenu du caractère généralement hydrophile des composés très volatils qui nous intéressaient, avons nous fait porter l'expérience sur le sérum obtenu par centrifugation du yoghourt $(2000 \mathrm{t} / \mathrm{mn}$ pendant $5 \mathrm{mn})$.

Cette modification n'a pas entraîné de changements notables pour les profils et la méthode s'est avérée très reproductible. Les figures 3 et 4 présentent les chromatogrammes obtenus par cette

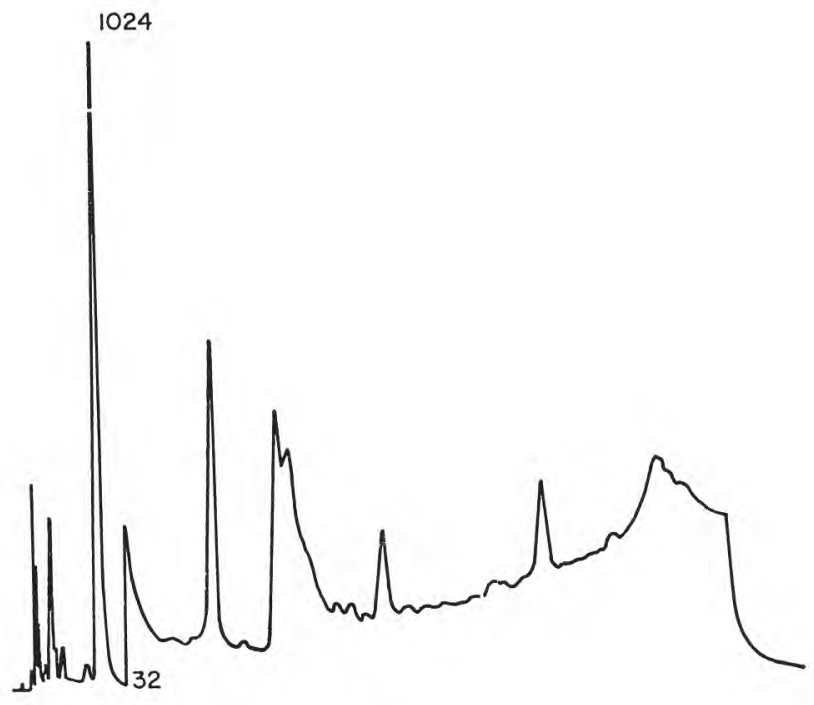

fig. 3

« Head Space » obtenu à partir du sérum d'un yoghourt fait avec du lait de vache (colonne 1)

technique à partir de yoghourts fabriqués dans les mêmes conditions, l'un avec du lait de vache et l'autre avec du lait de brebis.

Cependant, quel que soit l'échantillon utilisé (sérum ou yoghourt entier) une certaine fraction des composés volatils reste fixée sur les protéines. Cette observation déjà faite par Weurman [10] est confirmée par le chromatogramme (fig. 5) obtenu à partir d'un « yoghourt reconstitué » par dispersion dans l'eau distillée du culot de centrifugation correspondant au sérum analysé à la figure 4 . 


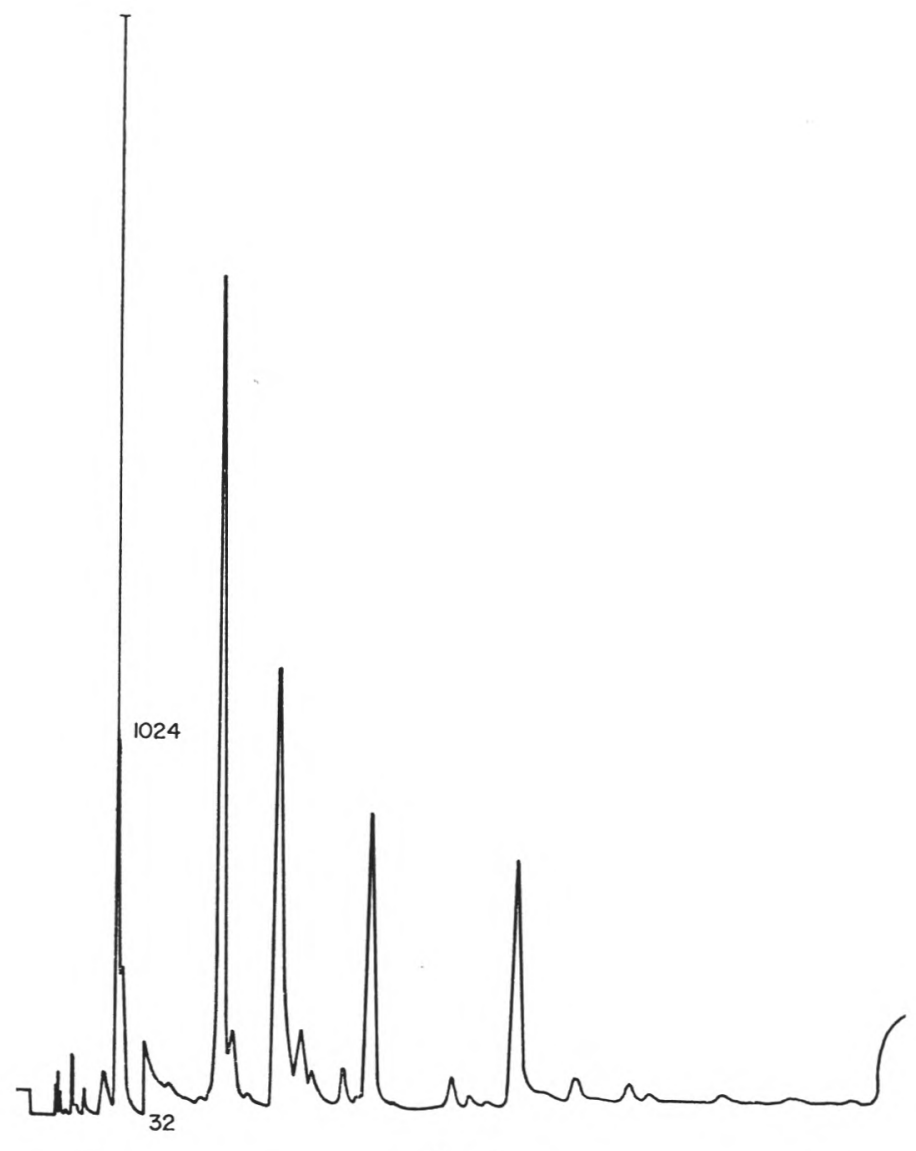

fig. 4

" Head Space » obtenu à partir du sérum d'un yoghourt fait avec du lait de brebis (colonne 1).

\section{C. - Mise en évidence de l'acétaldehyde dans le yoghourt de brebis}

1) Utilisation des temps de RÉTENTION RELATIFs

La comparaison des profils "vache-brebis » met en évidence une certaine similitude des 2 chromatogrammes. Pensant que le grand pic (atténuation 1024) présent dans les 2 cas pouvait correspondre à l'acétaldéhyde, nous avons déterminé, sur deux phases différentes, les temps de rétention relatifs par rapport à l'acétone des 2 grands pics ainsi que celui du produit de référence (tab. 2). 


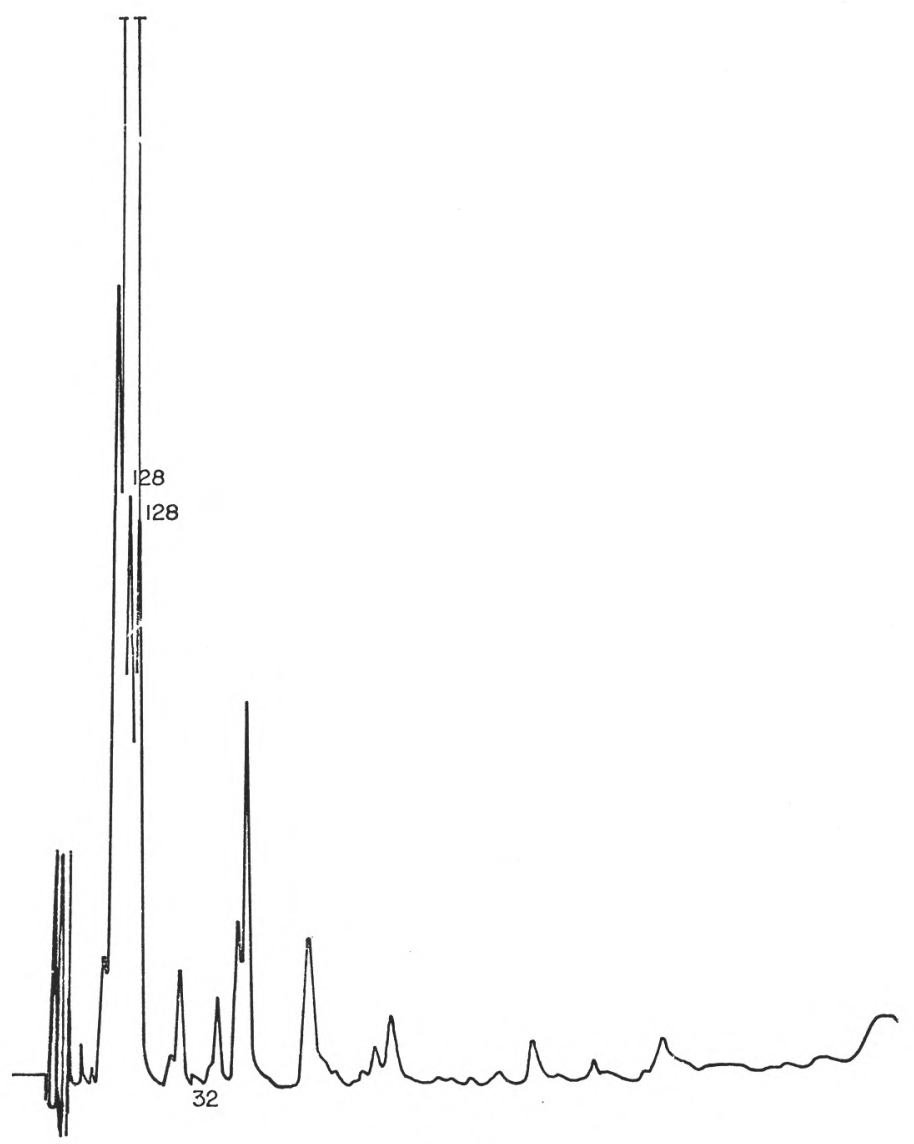

fig. 5

" Head Space » obtenu à partir de " yoghourt reconstitué » (colonne 1)

TABLEAU 2

\begin{tabular}{l|c|c|c}
\hline \multicolumn{1}{c|}{ Phase utilisée } & Vache & Brebis & Acétaldéhyde \\
\cline { 1 - 2 } & & & \\
Tricyano-éthoxypropane (colonne 1) & 0,34 & 0,36 & 0,35 \\
Porapak Q (colonne 2) & 0,42 & 0,42 & 0,42 \\
\hline
\end{tabular}

Volumes de rétention relatifs (par rapport à l'acétone), de l'acétaldéhyde et du pic majeur des sérums étudiés. 


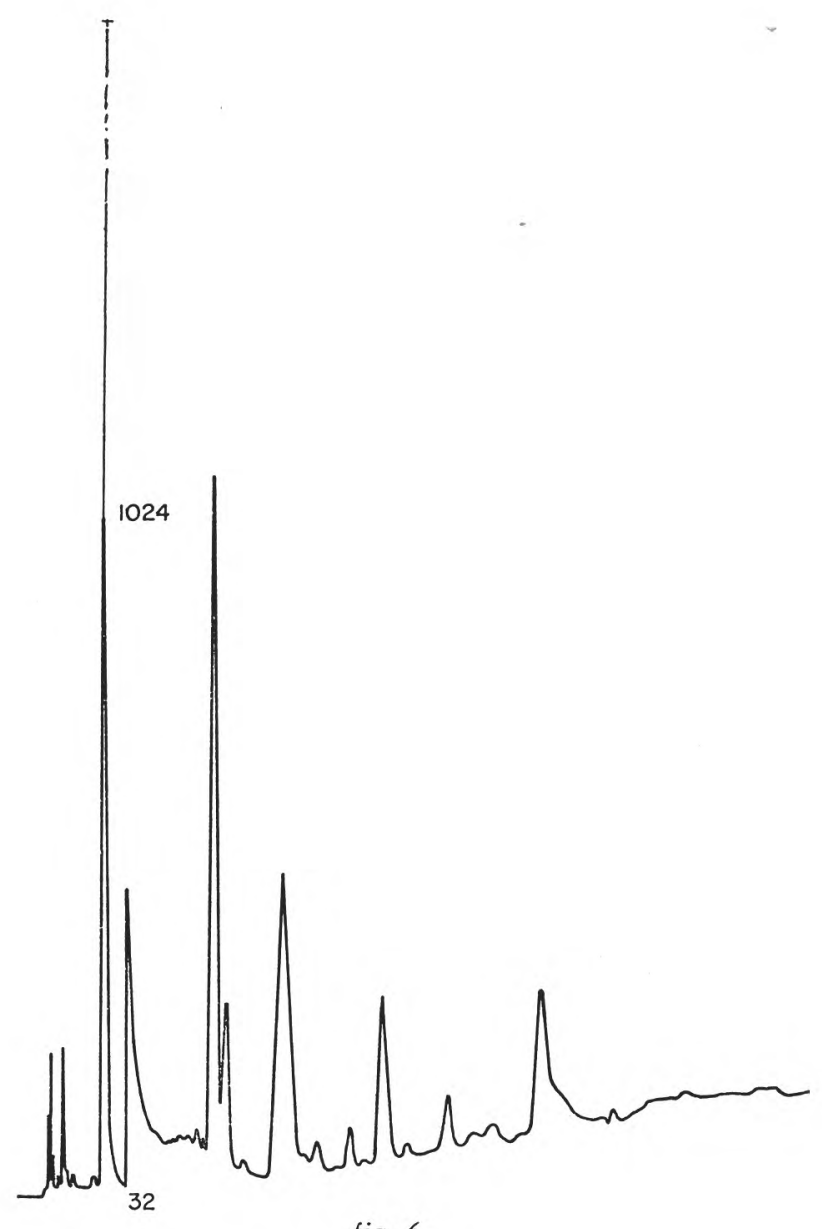

fig. 6

« Head Space » témoin obtenu à partir du sérum d'un yoghourt fait avec du lait de brebis (colonne 1)

2) ACTION DES RÉACTIFS DE LA FONCTION CARBONYLE (HYDROXYLAMINE ET BISULFITE DE SODIUM)

Si on compare un chromatogramme témoin (fig. 6) avec celui obtenu (fig. 7) à partir d'un échantillon du même sérum dans lequel on a ajouté, avant barbottage, quelques gouttes d'une solution aqueuse de chlorhydrate d'hydroxylamine, on constate que le grand pic soupçonné d'être l'acétaldéhyde a diminué très sensiblement ce qui permet de lui attribuer la fonction carbonyle et compte tenu, de la simi- 


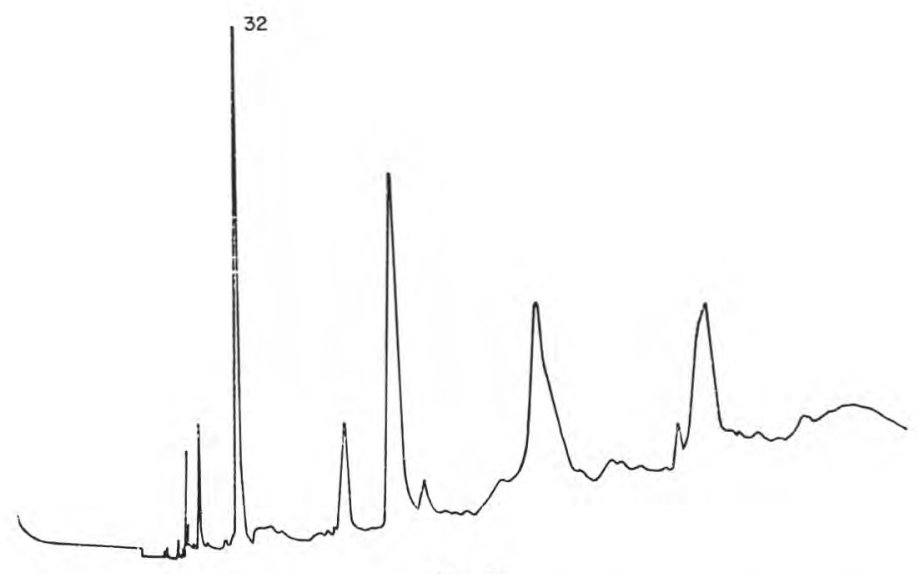

fig. 7

« Head Space » obtenu à partir du sérum précédent auquel on

a ajouté quelques gouttes d'une solution de chlorhydrate d'hydroxylamine (colonne 1)

litude des temps de rétention d'affirmer que l'on est en présence d'acétaldéhyde. La substitution de bisulfite de sodium à l'hydroxylamine conduit à la même conclusion.

\section{SYNTHESE - DISCUSSION}

La mise en évidence de la présence d'acétaldéhyde dans le yoghourt de lait de brebis à des concentrations voisines de celles auxquelles ce corps est présent dans le yoghourt fait avec du lait de vache vient en contradiction avec les observations de Schultz [7] sur lesquelles Turcic [11] s'est appuyé pour nier l'intérêt organoleptique de l'acétaldéhyde dans le yoghourt et étayer l'hypothèse selon laquelle les acides organiques seraient les composés essentiels de l'arôme. Il est très possible que la teneur élevée en matière sèche du yoghourt de brebis ait rendu délicat le dosage de l'acétaldéhyde par les procédés chimiques usuels.

Cependant, comme le yoghourt contient un certain nombre d'autres composés volatils et qu'il existe une disparité considérable entre les seuils de perception organoleptique des différentes substances chimiques, il est bien difficile d'évaluer la contribution individuelle de l'acétaldéhyde à l'arôme du yoghourt. La liste des corps que nous avons identifiés dans le yoghourt de vache est présentée dans le tableau 3 . 
TABLEAU 3

\begin{tabular}{l|l|l}
\hline \multicolumn{1}{c|}{ Composés } & Méthode d'isolement & Identification \\
& & \\
Acétaldéhyde & HS & TR \\
Acétone & HS & TR \\
Ethanol & HS & TR \\
Butanone & HS & TR \\
Diacétyle & HS-DVP & TR-SM \\
2 Pentanone & HS/DV & TR \\
Formate d'Ethyle & DVP & SM \\
Acétate d'Ethyle & DVP & SM \\
Isopropanol & DVP & SM \\
Benzène & DVP & SM \\
Chloroforme & DVP & SM \\
Acétoïne & DVP & SM \\
Ac Acétique & DVP & SM-IS \\
Ac Butyrique & DVP & IS \\
Ac Caproïque & DVP & IS \\
Ac Caprylique & DVP & IS \\
Ac caprique & DVP & \\
& & \\
\hline
\end{tabular}

HS = Head Space effectué sur yoghourt entier ou sur le sérum.

DVP = Distillation sous vide poussé du yoghourt entier.

HS/DV = Head Space effectué sur des extraits aqueux provenant de la distillation sous vide du yoghourt entier.

TR = Comparaison des temps de rétention.

$\mathrm{SM}=$ Spectrométrie de masse (couplage du chromatographe avec spectromètre Atlas $\mathrm{CH}$ 5).

IS $\quad=$ Identification spécifique $(12)+$ comparaison des temps de rétention.

\section{CONCLUSION}

L'efficacité satisfaisante de la méthode ainsi que le fait qu'une seule manipulation permette de mettre en évidence un grand nombre de corps dont le dosage s'effectue ordinairement de façon laborieuse laisse envisager diverses applications de la méthode décrite. En ce qui concerne le yoghourt il semble dans l'état actuel des connaissances qu'il faille voir dans l'acétaldéhyde un témoin intéressant du développement de l'arôme [13] sans négliger pour autant le rôle éventuel d'autres composants. 


\section{Remerciements}

Nous remercions MM. P. Dubois et J. Rigaut de la Station de Technologie Végétale de Dijon qui ont effectué les analyses par spectrométrie de masse.

Nous remercions $M . J: C l$. Vachot, de la Laiterie expérimentale, qui nous a fourni les échantillons de yoghourt utilisés au cours de cette étude.

\section{Ré s u m é}

Une méthode permettant l'analyse de substances volatiles présentes à très faible concentration est décrite. Son application à des yoghourts faits avec du lait de brebis a permis d'y mettre en évidence la présence, jusqu'alors contestée, d'acétaldéhyde à ces concentrations appréciables.

\section{S u $\mathbf{m} \mathbf{m}$ a $\mathbf{r} \mathbf{y}$}

This paper describes a trapping procedure for gas chromatography analysis of highly volatile flavor compounds. This method is suitable for yoghourt. It was possible, through its use, to demonstrate the presence of acetaldehyde in high concentration in yoghourt made with ews milk.

Reçu pour publication en juin 1972.

\section{Références bibliographiques}

[1] Weurman (C.) (1969). - J. Agr. Food Chem., 17 (2), 370-384.

[2] Teranishi (R.) et coll. (1971). - " Flavor Research ». Marcel Dekker Inc., New-York.

[3] Von Sydow (E.) et coll. (1970). - Lebensm. - Wiss.u. Techno., 3, 11-17.

[4] Morgan (M. E.) et Day (E. A.) (1965). - J. Dairy Sci., 48, 1382-1385.

[5] Dumont (J. P.) et AdDA (J.) (1972). - Le Lait, no 515-516, mai-juin, 311.

[6] Pette (J. W.) et Lolkema (H.) (1950). - Neth. Milk Dairy J., 4, 261-273.

[7] Schulz (M. E.), Voss (E.) et Kley (W.) (1954). - Milchwissenschaft, 9 (11), 361-365.

[8] Gorner (F.), Palo (V.) et Bertanova (M.) (1971). - Pol'nohospordantvo, 17, 378.

[9] Gorner (F.), Palo (V.) et Bertanova (M.) (1968). - Milchwissenschaft, 23 (2), 94-100.

[10] Weurman (C.) (1970). - Die Nahrung., 14 (7), 607-616.

[11] Turcic (M.), Rastic (J.) et Canic (V.) (1969). - Milchwissenschaft, 24 (5), 277-280.

[12] Mac Carthy (R. D.) et Duthie (A. H.) (1962). - J. Lipid Research., 3, (1), 117.

[13] Botrazi (V.) et Vescovo (M.) (1969). - Neth. Milk Dairy J., 23, 71-78. 